\title{
Salidroside Ameliorates Vascular Endothelial Cell Senescence through Downregulation of KLF4
}

\author{
Yanyan Zhang1,2*, Li He ${ }^{1 *}$, Mengxin Tu${ }^{1 *}$, Yongpan Huang3\#, Xiangchun Shen ${ }^{1 \#}$ \\ ${ }^{1}$ The Key Laboratory of Optimal Utilization of Natural Medicine Resources, School of Pharmaceutical Sciences, Guizhou Medical \\ University, Guiyang, China \\ ${ }^{2}$ Department of Clinical Pharmacy, School of Pharmaceutical Sciences, Guizhou Medical University, Guiyang, China \\ ${ }^{3}$ School of Medicine, Changsha Social Work College, Changsha, China \\ Email: "shenxiangchun@126.com, "yongpanhuangxy@163.com
}

How to cite this paper: Zhang, Y.Y., He, L., Tu, M.X., Huang, Y.P. and Shen, X.C. (2021) Salidroside Ameliorates Vascular Endothelial Cell Senescence through Downregulation of KLF4. Journal of Biosciences and Medicines, 9, 21-32.

https://doi.org/10.4236/jbm.2021.92003

Received: January 3, 2021

Accepted: February 7, 2021

Published: February 10, 2021

Copyright $\odot 2021$ by author(s) and Scientific Research Publishing Inc. This work is licensed under the Creative Commons Attribution International License (CC BY 4.0).

http://creativecommons.org/licenses/by/4.0/

\begin{abstract}
Salidroside is extensively used as a herbal medicine worldwide, and it has been shown to protect against disruption of endothelial homeostasis and act as an anti-aging agent. The present study aimed to investigate the ameliorative effects of salidroside on homocysteine (Hcy)-induced cell senescence in human umbilical vein endothelial cells (HUVECs) that were mediated via inhibition of Krüppel-like factor 4 (KLF4). An endothelial cell senescence model was induced by Hcy. The cell viability, activities of telomerase and lactate dehydrogenase $(\mathrm{LDH})$, and the level of reactive oxygen species were determined using commercial kits. The expression levels of KLF4, p53 and p21 were determined via western blot analysis, whereas the mRNA expression levels of KLF4 were detected by reverse transcription-quantitative PCR. Small interfering RNA-mediated knockdown of KLF4 was found to reverse Hcy-induced cell senescence. Hcy treatment led to an accelerated cell senescence, as evidenced by decreases in both cell viability and telomerase activity, whereas increases were noted in the leakage of LDH and the level of reactive oxygen species, in addition to an up-regulation of the protein levels of p53 and p21, and up-regulation of KLF4 at both the mRNA and protein level. Treatment with salidroside ameliorated Hcy-induced cell senescence in a dose-dependent manner. Taken together, these results suggested that Hcy may induce cell senescence through upregulation of KLF4, and this may be reversed by treatment with salidroside. Therefore, salidroside was shown to inhibit Hcy-induced cell senescence through KLF4 inhibition.
\end{abstract}




\section{Keywords}

Cell Senescence, Salidroside, HUVECs, Human Umbilical Vein Endothelial

Cells, Krüppel-Like Factor 4, KLF4, Homocysteine

\section{Introduction}

With the rapid increase in an aging population that is currently underway, aging-associated diseases, including cardiovascular diseases (CVDs), pose serious threats to human life and health [1]. Among the CVDs, atherosclerosis (AS) provides the pathological basis of cardiovascular events. Although its pathogenesis has yet to be fully elucidated, AS is best categorized as a chronic vascular inflammatory disease [2]. Homocysteine (Hcy) is an intermediate product of methionine metabolism in vivo, and is closely associated with cardiovascular events, considered as an independent risk factor for AS [3] [4] [5] [6]. As an inflammatory stimulant, Hcy affects the function of endothelial cells and smooth muscle cells. It is also involved in oxidative stress and inflammatory reactions, and effects change both in gene expression activity and in other mechanisms, leading to the occurrence and development of AS [7]. Oxidative stress caused by high levels of Hcy is closely associated with aging-related diseases, including AS and diabetes mellitus [8] [9]. Patients with severely high levels of Hcy undergo an accelerated senescence, and a series of symptoms associated with senescence typically appear during the early stage of life.

The KLF family proteins comprise a conserved zinc-finger transcription factor family in eukaryotes, and KLF4 is an important member of the KLF family. It contains two transcriptional activation domains and transcription inhibitory domains at different sites, which can be used, respectively, as transcriptional activators and inhibitors of genes associated with cell proliferation, differentiation and apoptosis [10]. Recently, it has been demonstrated that KLF4 is an important transcription factor involved in regulating the cardiovascular system [11], which can inhibit or activate inflammatory factors, and variously regulate the activation of vascular endothelial cells, the polarization of macrophages, and the proliferation and migration of smooth muscle cells through different pathways to exert cardiovascular protection [12]. Previous studies have shown that KLF4 is able to inhibit the proliferation of smooth muscle cells by regulating the expression of p53- and p21-associated genes in the cell cycle [13]. Overexpression of KLF4 can induce both the down-regulation of anti-aging gene expression and the up-regulation of cell senescence genes. These findings suggest that KLF4 induces cell senescence, and that the transcription factor KLF4 may have an important role in the mechanism of endothelial senescence.

Rhodiola is a perennial herb or subshrub of the genus Rhodiola in the family Rhodiola that is used in the treatment of asthma, chronic obstructive emphysema, influenza, and so on. Salidroside is the main active component of Rhodiola, 
and it possesses extensive pharmacological activity, especially in anti-aging and anti-oxidation processes [14]. Numerous modern pharmacological studies of salidroside have confirmed that the compound is a characteristic drug resource, which affords vascular endothelial protection, regulates and improves endothelial homeostasis, and intervenes in the regulation of aging, although its mechanism of action has yet to be fully elucidated. In the present study, the molecular mechanism by which salidroside ameliorates endothelial aging was investigated through an analysis of Hcy-induced senescence of vascular endothelial cells, and the effect of salidroside on the regulatory transcription factor KLF4 during the pathological process of endothelial aging was also explored in order to clarify its regulatory and protective effects on vascular endothelial function.

\section{Materials and Methods}

Isolation, culture and subculture of human umbilical vein endothelial cells (HUVECS). We followed the methods of Zhang et al. 2020 [15]. Umbilical vein endothelial cells (HUVECs) were purchased from The Cell Bank of Type Culture Collection of the Chinese Academy of Sciences. Cells were cultured in Endothelial Cell Medium (ScienCell Research Laboratories, Inc.) supplemented with 5\% FBS (ScienCell Research Laboratories, Inc.) and $100 \mathrm{U} / \mathrm{ml}$ penicillin and streptomycin, and maintained in an incubator at $37^{\circ} \mathrm{C}$ with $5 \% \mathrm{CO}_{2}$. Cells were subcultured and seeded into 6- or 24-well plates for subsequent experiments, as detailed below. The cells were pretreated with salidroside for $2 \mathrm{~h}$ at $37^{\circ} \mathrm{C}$, Hcy (PeproTech EC Ltd.) was subsequently added, and the cells were incubated for a further $72 \mathrm{~h}$. HUVECs were trypsinized with $0.25 \%$ trypsin and collected for analysis.

Reagents. DMEM and fetal bovine serum (FBS) were purchased from Nanjing BioChannel Biotechnology Co., Ltd. RNA extraction kit was obtained from Qiagen $\mathrm{GmbH}$. Invitrogen ${ }^{\circledR}$ Lipofectamine $^{\mathrm{TM}} 2000$ transfection reagent was from Thermo Fisher Scientific, Inc., and polyvinylidene fluoride (PVDF) membranes were purchased from EMD Millipore, whereas electrochemiluminescence (ECL) Plus ${ }^{\circledR}$ Western blotting reagent and horseradish peroxidase (HRP)-coupled secondary antibodies were from ImmunoWay Biotechnology Company.

Determination of cell activity by MTT assay. The cultured cells were digested and completely suspended in solution. Subsequently, the cells were used to inoculate a 96-well plate (cell density, $1.5 \times 10^{4}$ cells/well) and then treated with different factors for 2 days (once in the middle of the plate) $24 \mathrm{~h}$ later. MTT solution $(20 \mu \mathrm{l} ; 5 \mathrm{mg} / \mathrm{ml})$ was subsequently added to each pore in $200 \mu \mathrm{l}$ culture medium. After $4 \mathrm{~h}$, the supernatant was carefully absorbed and discarded, and $150 \mu \mathrm{l}$ DMSO was added to each pore with agitation for $10 \mathrm{~min}$, prior to reading the absorbance value of each well at a wavelength of $570 \mathrm{~nm}$ by enzyme-linked immunosorbent assay. Each treatment group was performed in triplicate.

Assessment of cell senescence using $\beta$-galactosidase ( $S A$ - $\beta$-gal) staining. SA- $\beta$-gal staining was performed using a Cellular Senescence Assay kit (CBA-230; Cell Biolabs Inc.) according to the manufacturer's protocol with minor modifica- 
tions. The HUVECs were plated in 6-well plates at a density of $3 \times 10^{5}$ cells/well and then incubated overnight, fixed, and the cells that were stained for SA- $\beta$-gal were photographed using a camera that was attached to a light microscope (FSX100; Olympus Corporation) to assess the extent of cell senescence. Areas measuring $0.44 \times 0.32 \mathrm{~mm}$ (length $\times$ width) from each image were scanned. For the image preparation, type "8-bit" was selected, and the "adjust," "threshold max 180", and "measure" commands in ImageJ software (National Institutes of Health) were used. The results of "\% area" were used to represent the staining level of SA- $\beta$-gal.

Determination of the levels of intracellular oxygen free radicals. The fluorescence dye DCFH-DA, which in itself has no fluorescence, can freely pass through the cell membrane and enter cells. After having entered the cell, the dye is hydrolyzed by an esterase in the cell to form DCFH. DCFH is not able to penetrate the cell membrane, and the reactive oxygen species (ROS) in the cells are able to oxidize the non-fluorescent DCFH to form the green fluorescent product, DCF. Therefore, the level of intracellular DCF fluorescence can be used as a marker to reflect the amount of ROS present. For this assay, the cells were initially cultured in a 6-well plate for $48 \mathrm{~h}$. The cell culture medium was subsequently removed, the cells were rinsed three times in serum-free medium at room temperature, $10 \mu \mathrm{M}$ DCFH-DA fluorescent dye was added, and the cells were then incubated at $37^{\circ} \mathrm{C}$ for a further $20 \mathrm{~min}$. The cells were subsequently washed three times with serum-free medium, and the fluorescence intensity was measured using a multi-function plate reader.

Determination of telomerase activity by reverse transcription-quantitative $P C R(R T-P C R)$. Telomerase activity in the extract was detected after the living cells had been lysed. Under certain buffer conditions, telomerase can synthesize telomere repeats by using dNTPs at the end of the oligonucleotides. Following RT-qPCR amplification of the telomerase amplification product, SYBR Green, which is a nucleic acid stain, can be used to detect the fluorescence signal of the amplification product. In this manner, the telomerase activity was measured using the RT-qPCR method, and the activity of telomerase was calculated.

Detection of changes in the mRNA level using $R T-q P C R$. The total RNA was extracted from cells using TRIzol ${ }^{\circledR}$ reagent, and the amount of RNA was quantified using a NanoDrop ND-2000 spectrophotometer (Thermo Fisher Scientific, Inc.). The RNA was reverse-transcribed using reverse transcriptase, and subsequently the miR-24/U6 and E2F1/GAPDH levels were quantified using a CFX96 Real-Time PCR detection system (Bio-Rad Laboratories, Inc.). The primer sequences used for RT-qPCR are shown in Table 1.

Western blotting. At $2 \mathrm{~h}$ after reperfusion, the heart tissues were harvested. The total protein was extracted with RIPA lysis buffer from ischemic heart tissues, transferred onto a PVDF membrane (EMD Millipore), sealed with 5\% skimmed milk powder, and incubated with the anti-E2F2 primary antibody (Cell Signaling Technology, Inc.) overnight. The membranes were then incubated again with the HRP-coupled secondary antibody (ImmunoWay Biotechnology 
Table 1. Primers used for the reverse transcription-quantitative PCR experiments.

\begin{tabular}{cc}
\hline Gene name & Primer or gene sequence \\
\hline \multirow{2}{*}{ KLF4 } & Upstream: 5'-TCAAGGGAGCACGCTATGTCT-3' \\
& Downstream: 5'-CCTCTTCTTGCCGCTTCAGT-3' \\
Mouse GAPDH & Upstream: 5'-AACTTTGGCATTGTGGAAGG-3' \\
& Downstream: 5'-TGTGAGGGAGATGCTCAGTG-3' \\
\hline
\end{tabular}

Company), followed by analysis using an ECL system (Fusion FX7 imaging system; Vilber China).

Statistical analysis. Statistical Product and Service Solutions (SPSS) 21.0 software (IBM Corp.) was used for statistical analysis. Numerical variables were expressed as mean \pm standard deviation (SD), and independent-samples t-test was adopted for comparisons between two groups. $\mathrm{P}<0.05$ was considered to indicate a statistically significant difference.

\section{Results}

Determination of cell activity. After having treated the cells with $500 \mu \mathrm{M}$ Hcy for $48 \mathrm{~h}$, the viability of the endothelial cells was significantly decreased (Figure 1(A) and Figure 1(B)). Salidroside administration significantly increased cell viability in a concentration-dependent manner. $N$-acetylcysteine (NAC) elicited the same effect (Figure $1(\mathrm{C})$ ). The extent of damage caused to the cell membrane was subsequently measured by LDH leakage. The leakage amount of LDH increased significantly after $48 \mathrm{~h}$ of Hcy treatment, whereas salidroside was able to reduce the leakage to differing degrees (Figure 1(D)). Collectively, these results demonstrated that salidroside exerted a protective effect on Hcy-induced endothelial cell injury.

Determination of intracellular ROS. Endothelial cells exposed to high concentrations of oxidative stress for a long period of time undergo damage and dysfunction. In order to evaluate the inhibitory effect of salidroside on the production of ROS induced by Hcy, DCFH-DA fluorescence probe was used to detect the ROS level. Incubation of the cells with Hcy for $48 \mathrm{~h}$ led to significant increases in the levels of ROS, although the ROS levels were significantly decreased following treatment with salidroside (Figure 2(A)). The statistical results of fluorescence intensity detection were found to be consistent (Figure 2(B)).

Effect of salidroside on cell senescence. To investigate cell senescence under Hcy-induced conditions, the percentage of positive cells was detected by SA- $\beta$-gal staining. As shown in Figure 3(A) and Figure 3(B), the number of positively stained cells increased significantly following Hcy treatment, whereas the number of positively stained cells decreased after salidroside intervention at the different applied concentrations. The statistical results of the percentages of positively stained cells were found to be consistent.

Telomerase activity is closely associated with cell senescence. RT-qPCR was performed to detect the levels of telomerase activity. As shown in Figure 3(C), 


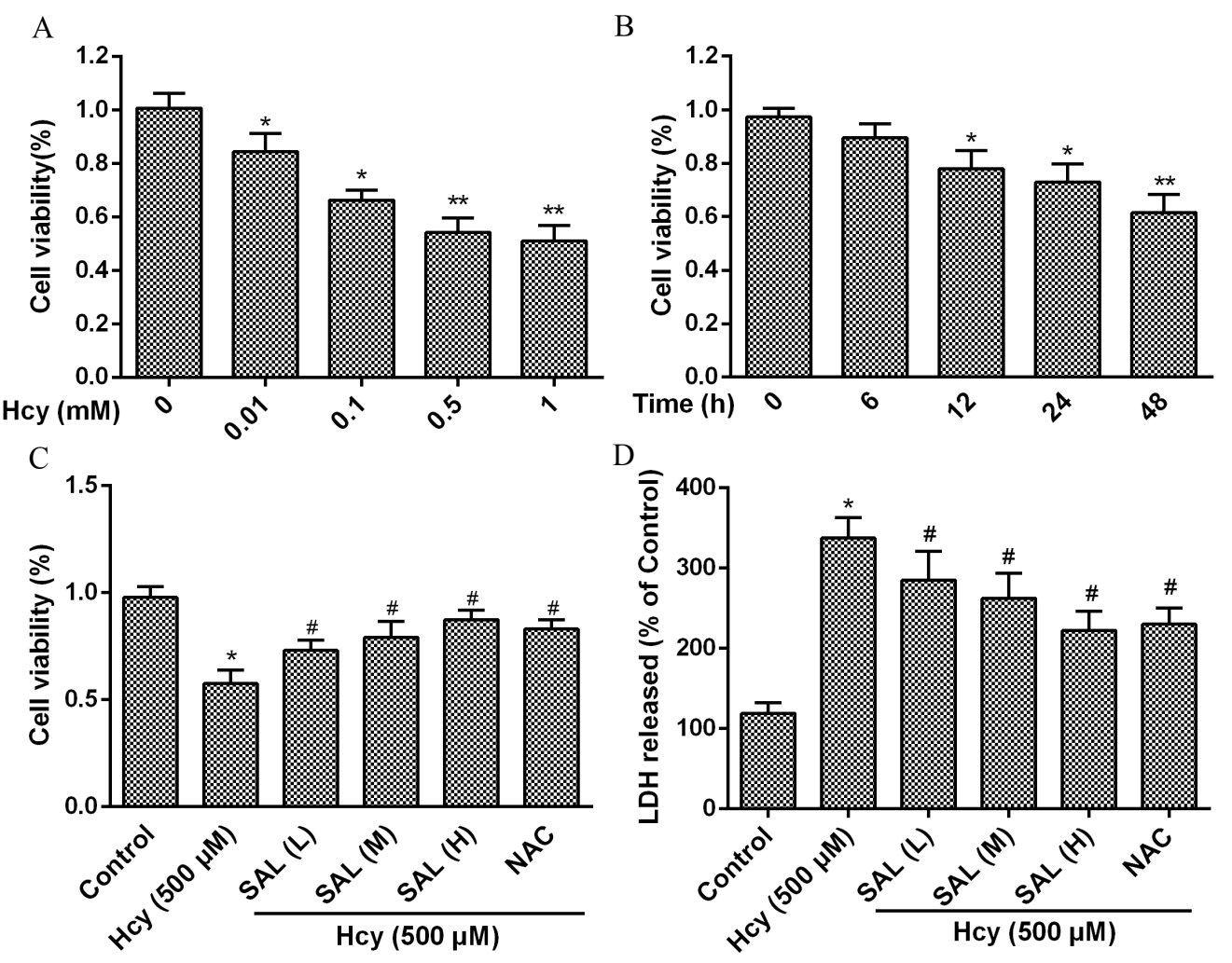

Figure 1. Salidroside increases endothelial cell viability and decreased LDH leakage. (A) Effect of $0.01,0.1,0.5$ and $1 \mathrm{mM}$ Hcy on cell viability is shown. (B) Effect of $500 \mu \mathrm{M}$ Hcy on cell viability at 6, 12,24 and $48 \mathrm{~h}$ is shown. (C) Effect of salidroside treatment on the cell viability induced by $500 \mu \mathrm{M}$ $\mathrm{Hcy}$ for $48 \mathrm{~h}$ is shown ( $\mathrm{N}$-acetylcysteine was used as the positive drug control). (D) Effect of salidroside on lactate dehydrogenase leakage. Bars represent the mean \pm SD for three independent experiments. ${ }^{\star} \mathrm{P}<0.05,{ }^{*} \mathrm{P}<0.01$ vs. the Control group; ${ }^{\sharp} \mathrm{P}<0.05$ vs. Hcy group. Hcy, homocysteine.
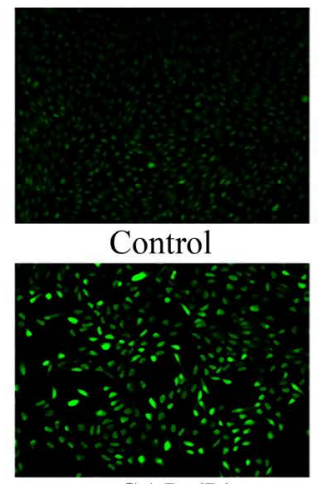

SAL $(\mathrm{L})$

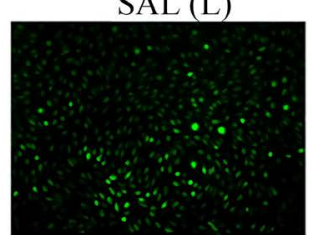

SAL $(\mathrm{H})$

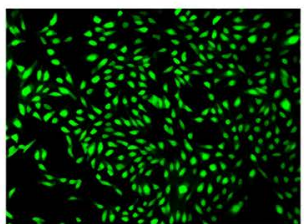

$\mathrm{Hey}$

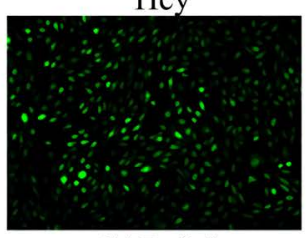

SAL (M)

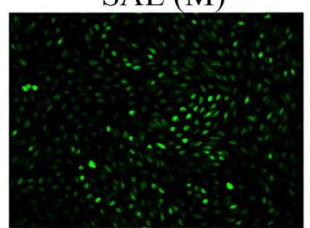

NAC

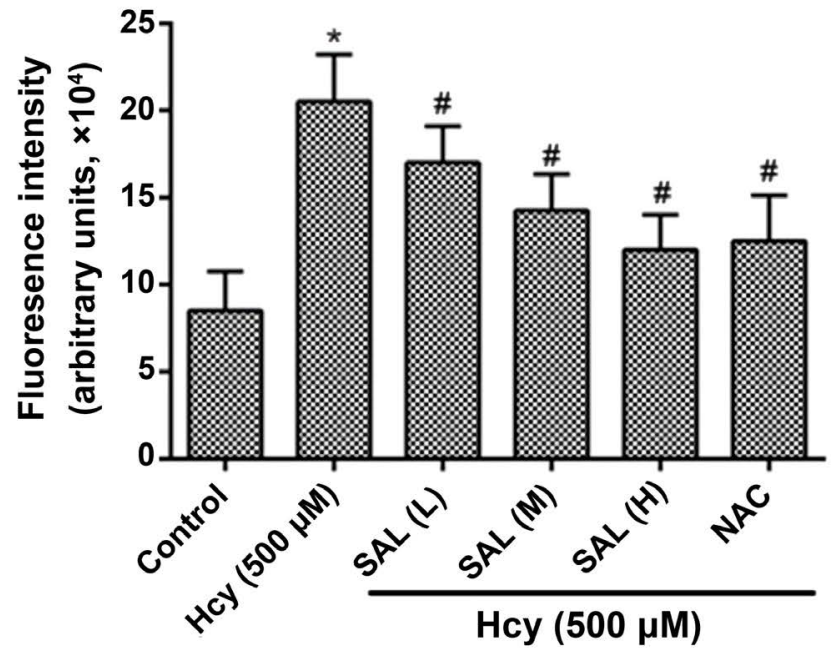

Figure 2. Salidroside decreases the levels of ROS in endothelial cells (as shown by the green color; magnification, $\times 200$ ). Bars represent the mean \pm SD for three independent experiments. ${ }^{*} \mathrm{P}<0.01$ vs. the Control group; ${ }^{*} \mathrm{P}<$ $0.05,{ }^{* \#} \mathrm{P}<0.01$ vs. Hcy group. Hcy group. Hcy, homocysteine; ROS, reactive oxygen species. 
A
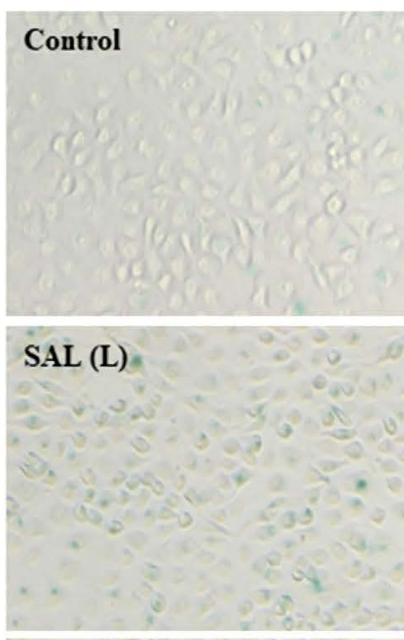

SAL (H)
Hcy

SAL (M)

NAC
B

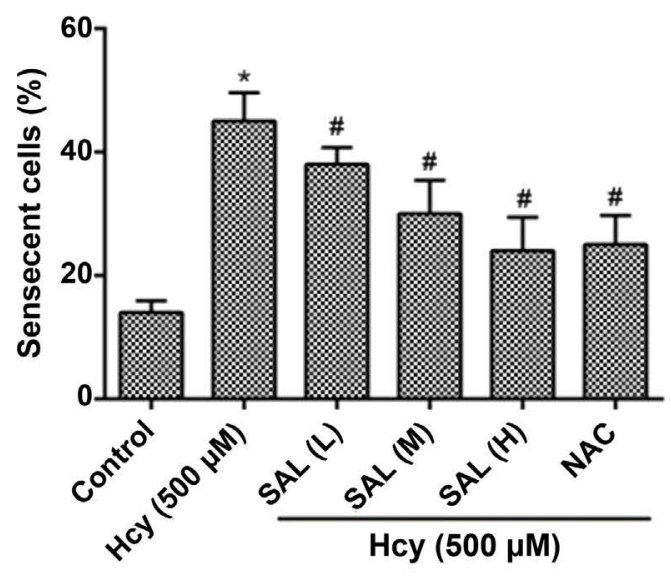

C

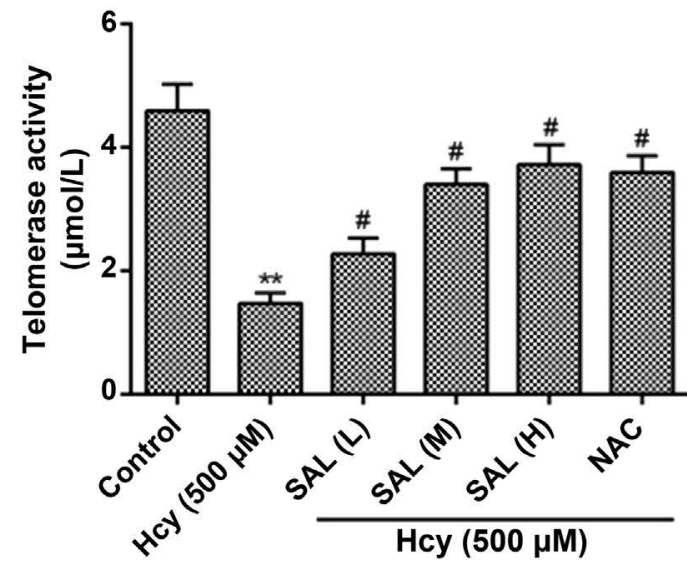

Figure 3. Salidroside inhibits Hcy-induced endothelial cell senescence. (A) SA- $\beta$-gal staining of HUVECs treated with Hcy and salidroside are shown by the blue coloration (magnification, $\times 200$ ). (B) Quantitative analysis of the SA- $\beta$-Gal positively stained cells. (C) Telomerase activity was detected by reverse transcription-quantitative PCR after $48 \mathrm{~h}$ treatment with Hcy and salidroside. Bars represent the mean $\pm \mathrm{SD}$ for three independent experiments. ${ }^{\star} \mathrm{P}<0.05,{ }^{\star \star} \mathrm{P}<0.01$ vs. the Control group; ${ }^{\sharp} \mathrm{P}<0.05$, ${ }^{\# \#} \mathrm{P}<$ 0.01 vs. Hcy group. Hcy, homocysteine; SA- $\beta$-gal, $\beta$-galactosidase; HUVECs, human umbilical vein endothelial cells.

Hcy caused a significant decrease in telomerase activity, whereas salidroside administration markedly increased telomerase activity. These results suggested that salidroside could significantly inhibit the Hcy-induced senescence of endothelial cells.

Effect of salidroside on the expression of senescence-associated molecular markers ( $p 53$ and p21) in Hcy-induced endothelial cell senescence. p53 and p21 are considered as the important biomarkers in cell senescence. To investigate the effects of salidroside on the expression of p53 and p 21 in Hcy-induced endothelial cell senescence, western blotting was utilized to assess the expression levels of p53 and p21. As shown in Figure 4, compared with the control group, Hcy treatment led to increases in the protein expression levels of p53 and p21. However, salidroside intervention significantly reduced the expression levels of p53 and p21. These results indicated that salidroside was able to exert a protective effect on Hcy-induced endothelial cell senescence.

KLF4 expression during endothelial cell senescence. As previously reported, KLF4 is an evolutionarily conserved zinc finger-containing transcription factor 

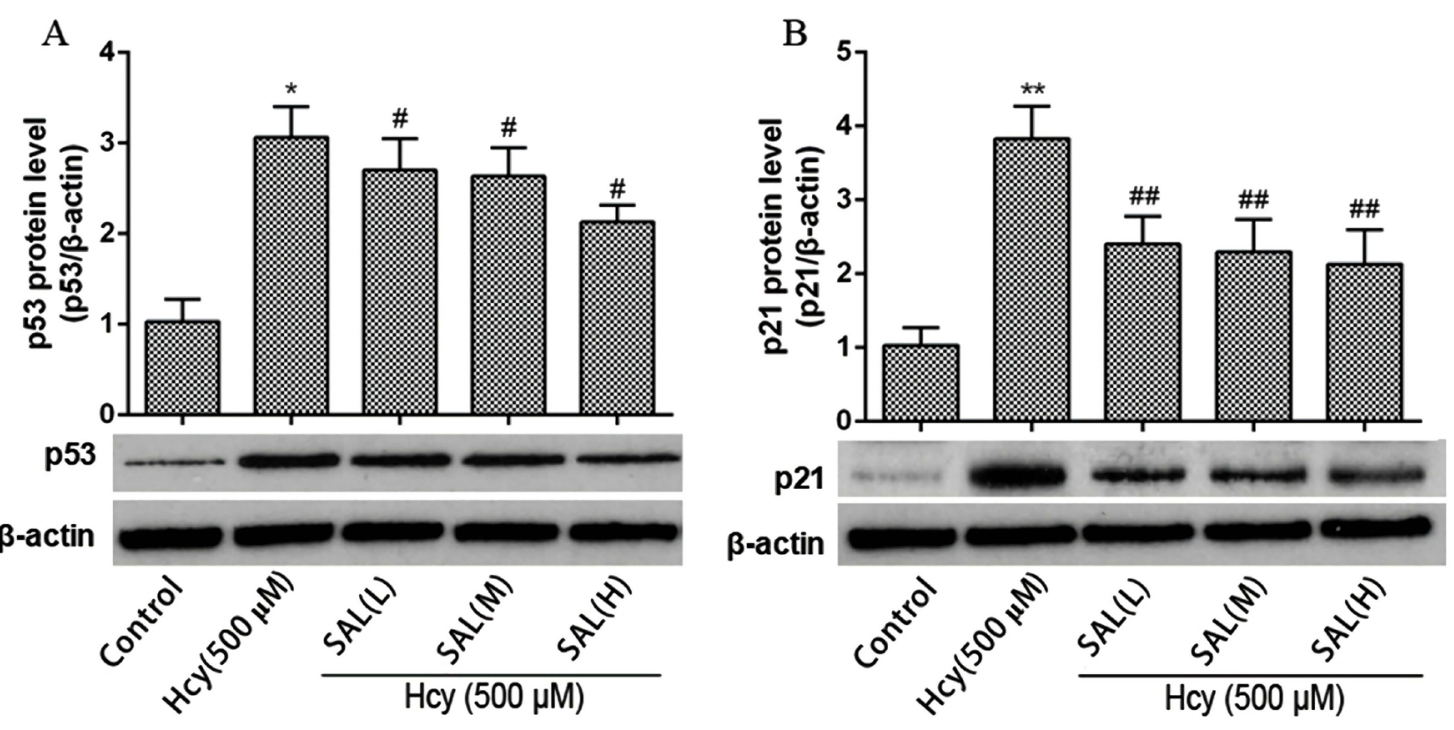

Figure 4. Salidroside can down-regulate the protein expression of p53 and p21 genes. Expression levels of (A) p53 and (B) p21 were determined using western blot analysis. Bars represent the mean \pm SD for three independent experiments. ${ }^{\star} \mathrm{P}<0.05,{ }^{\star *} \mathrm{P}<0.01$ vs. the Control group; ${ }^{\sharp} \mathrm{P}<0.05$ vs. Hcy group. Hcy, homocysteine.

that is involved in various cellular processes, including maintaining intestinal epithelial homeostasis, proliferation, migration and tube formation in human retinal microvascular endothelial cells. In order to determine whether KLF4 is involved in Hcy-induced endothelial cell senescence, western blotting and RT-qPCR were used to assess the expression levels of KLF4. As shown in Figure 5, Hcy up-regulated the expression of KLF4 at both the protein and the mRNA levels in endothelial cell senescence. Additionally, salidroside led to reductions in the mRNA and protein levels of KLF4 in a concentration-dependent manner. These results suggested that KLF4 is involved in the senescence of endothelial cells, and salidroside was able to inhibit Hcy-induced cell senescence via up-regulation of KLF4.

Knockout of KLF4 attenuates the senescence of endothelial cells induced by Hcy. To investigate the association between salidroside and KLF4, a KLF4-specific siRNA was utilized to knock down the expression of KLF4. As shown in Figure 6(A), the western blot analysis demonstrated that the expression of KLF4 was significantly reduced following transfection with KLF4 siRNA. As presented in Figure 6(B), KLF4 expression was upregulated in the Hcy group, and its expression level was significantly repressed when exposed to siKLF4. The percentage of positive cells was subsequently detected by SA- $\beta$-gal staining. The results showed that the number of positive cells in the model group was significantly higher compared with that in the normal group, whereas the number of positive cells in the KLF4 siRNA transfection group was significantly lower compared with that in the model group (Figure 6(C)). These results suggested that KLF4 is closely associated with the aging process of endothelial cells induced by Hcy, and the degree of endothelial cell senescence induced by Hcy can be ameliorated by knocking down KLF4. 
A

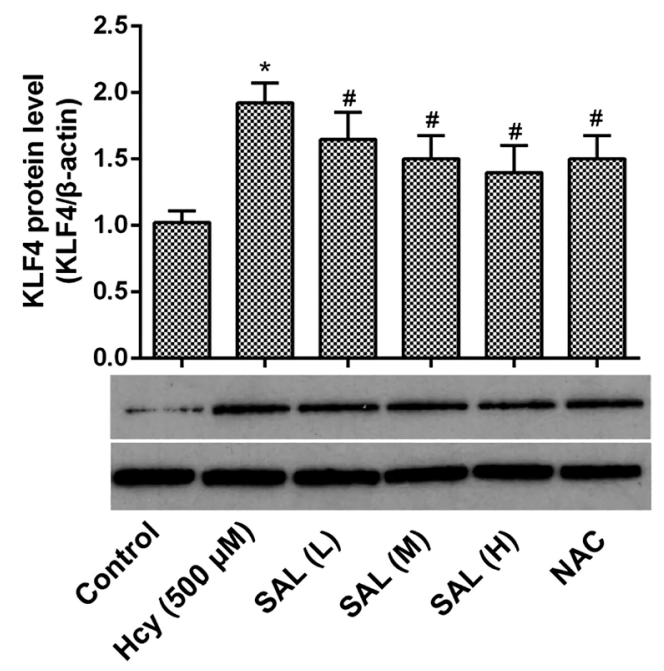

B

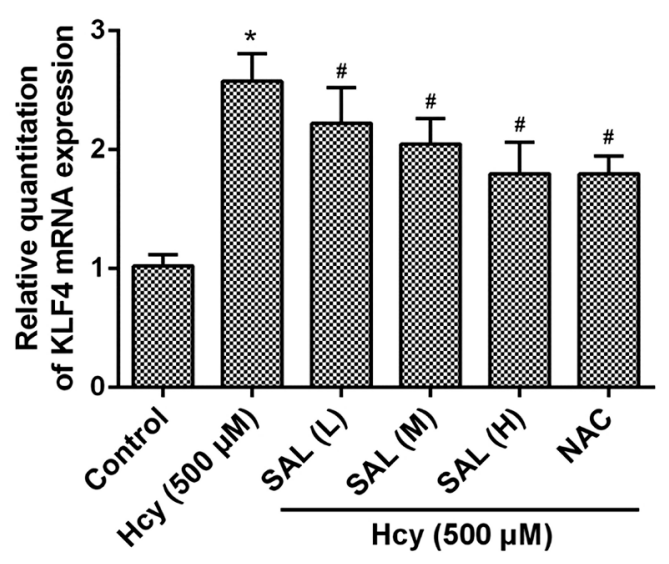

Figure 5. Salidroside can down-regulate the protein and mRNA levels of KLF4. (A) Protein expression levels of KLF4 were determined using western blot analysis, whereas (B) mRNA expression levels of KLF4 were determined using reverse transcription-quantitative PCR. Bars represent the mean \pm SD for three independent experiments. ${ }^{\star} \mathrm{P}<0.05$ vs. the Control group; ${ }^{*} \mathrm{P}<0.05$ vs. Hcy group. Hcy, homocysteine; KLF4, Krüppel-like factor 4.

A
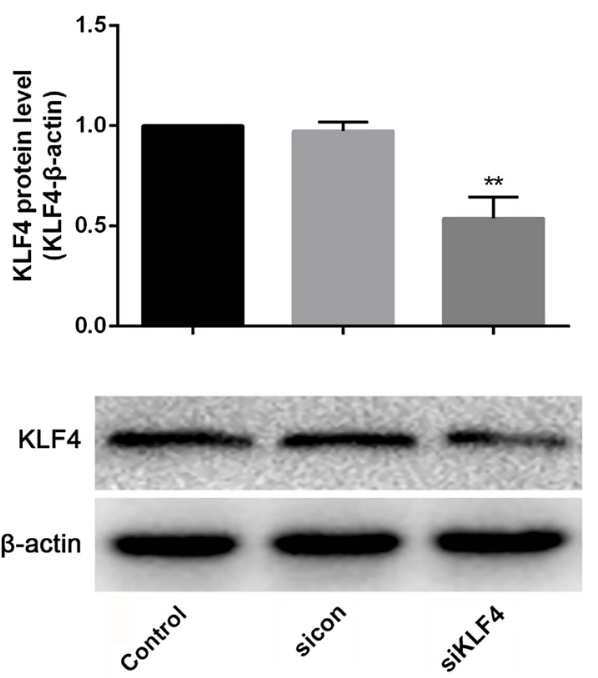

$\mathrm{B}$
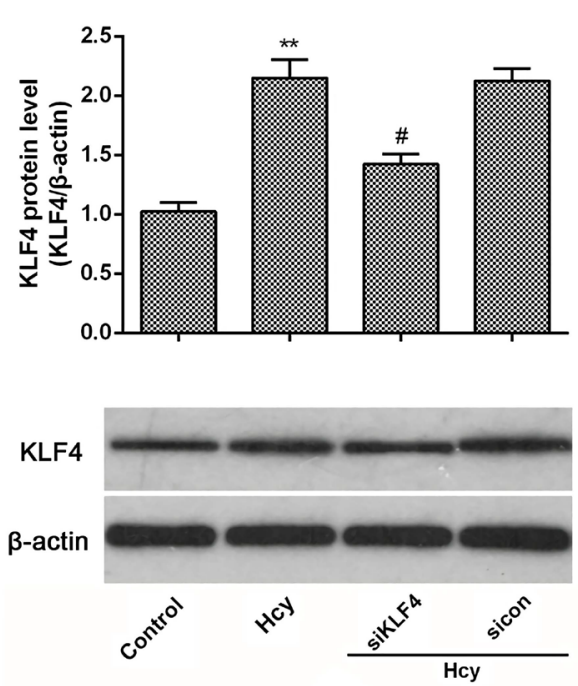

C
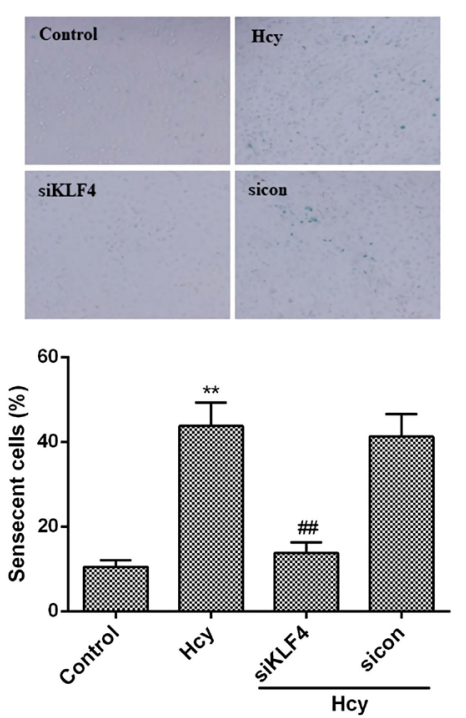

Figure 6. Silencing KLF4 is able to significantly inhibit the senescence of endothelial cells induced by Hcy. (A) The efficacy of siKLF4 transfection was determined by western blotting. (B) Detection of KLF4 protein expression in HUVECs after KLF4 siRNA treatment, as measured by western blotting. (C) SA- $\beta$-gal staining of HUVECs treatment with Hcy and siKLF4, as shown by the blue coloration (magnification, $\times 200$ ), and quantitative analysis of SA- $\beta$-Gal-positive cells. Bars represent the mean \pm SD for three independent experiments. ${ }^{\star} \mathrm{P}<0.05,{ }^{\star \star} \mathrm{P}<0.01$ vs. the Control group; ${ }^{\#} \mathrm{P}<0.05,{ }^{* \#} \mathrm{P}<0.01$ vs. Hcy group. Hcy, homocysteine; SA- $\beta$-gal, $\beta$-galactosidase; HUVECs, human umbilical vein endothelial cells; KLF4, Krüppel-like factor 4.

\section{Discussion}

Vascular aging is an important process, causing a high mortality rate due to CVDs. Vascular endothelial cells are one of the main components of the vascular wall, and they contribute significantly to the biological processes of vascular ag- 
ing. Changes in endothelial function occur at an early stage during vascular aging. As an early pathophysiological change, vascular endothelial dysfunction caused by aging has an important role in the occurrence and development of CVDs [16]. Therefore, a study of the mechanisms of aging that underpin endothelial cell function provides the basis for the overall study of vascular aging. The study of endothelial dysfunction and endothelial protection is also helpful for the purposes of delaying vascular aging, and to reduce both the incidence of CVDs and mortality rates associated with CVDs.

Transcription factors have a central role in the regulation of various physiological processes. In the present study, the role of the transcription factor KLF4 in Hcy-induced endothelial cell senescence was investigated. KLF4 is an important member of the KLF transcription factor family. As a transcription factor, it activates or inhibits the expression of multiple genes, and participates in the regulation of cell proliferation, mediating cell differentiation and apoptosis. Studies have shown that overexpression of KLF4 may induce cell senescence during the process of inducing pluripotent stem cells, wherein cell senescence is induced by up-regulating p21 expression. In addition, using a miRNA chip, a series of different miRNA species that may be regulated by KLF4 and could be involved in cell senescence regulation were identified, and it was shown that KLF4 may be involved in cell senescence via promoting the expression of miR-203 [17]. The data in the present study revealed that the expression of KLF4 in an endothelial cell aging model induced by Hcy was up-regulated at both the protein and mRNA levels. After having constructed the lentivirus to achieve knockout of KLF4, the results of SA- $\beta$-gal staining revealed that the number of positively stained cells decreased significantly. It was thereby further confirmed that KLF4 is closely associated with the senescence of endothelial cells induced by Hcy, and a low level of KLF4 leads to an attenuation of endothelial cell senescence.

Salidroside is a natural phenolic secondary metabolite existing in Rhodiola plants, and it has been shown to exert significant anti-aging and antioxidant pharmacological effects. It has been shown that salidroside is able to reduce lipid deposition in HUVECs, down-regulate the expression of aging-associated molecular markers (p66, p53 and p21), and delay cell senescence [18]. In addition, Mao et al. [19] found that salidroside has a role in changing the morphology and cell cycle of cells induced by oxidation, as well as the expression of p21 gene, a cell cycle-dependent kinase inhibitor and showed that salidroside, in an effective preparation, was able to delay human aging and to reduce the incidence of aging-associated diseases. In the present study, Hcy induced endothelial cell injury and senescence, led to a marked increase in cell viability and telomerase activity, and decreased LDH leakage and ROS levels following treatment with salidroside. At the same time, the protein expression levels of p53 and p 21 were significantly decreased. Taken together, these results suggested that salidroside has an important role in inhibiting Hcy-induced endothelial cell senescence. In addition, the expression of KLF4 at both the protein and the mRNA levels was down-regulated in the salidroside treatment group, thereby consolidating the evidence for the 
involvement of KLF4 in the inhibition of Hcy-induced endothelial cell senescence by salidroside.

\section{Conclusion}

In conclusion, in the present study salidroside has been found to ameliorate the endothelial cell senescence induced by Hcy, and to down-regulate the expression of the transcription factor KLF4 at the protein and mRNA levels. Additionally, reducing the level of KLF4 via knockdown of the protein attenuated Hcy-induced endothelial cell senescence, indicating that the protective effect of salidroside on Hcy-induced endothelial cell senescence was mediated via regulation of the transcription factor KLF4. Taken together, these results suggest that KLF4 is a potential target for the study of vascular aging and other aging-associated diseases, although the precise mechanism by which salidroside regulates KLF4 in protecting against endothelial cell senescence requires further study.

\section{Funding}

This work was supported by the National Natural Science Foundation of China (U1812403-4-4), the Scientific and Technological Founding of Guizhou Province [grant no. QKHJ (2016)1128], the International Scientific and Technological Cooperation Base of Guizhou Province [grant no. 2017-5802] and Guizhou Provincial Key Technology R\&D Program [grant no. (2020)4Y093].

\section{Conflicts of Interest}

The authors declare no conflicts of interest.

\section{References}

[1] Vigen, R., Maddox, T.M. and Allen, L.A. (2012) Aging of the United States Population: Impact on Heart Failure. Current Heart Failure Reports, 9, 369-374. https://doi.org/10.1007/s11897-012-0114-8

[2] Lahoute, C., Herbin, O., Mallat, Z., et al. (2011) Adaptive Immunity in Atherosclerosis: Mechanisms and Future Therapeutic Targets. Nature Reviews Cardiology, 8 , 348-358. https://doi.org/10.1038/nrcardio.2011.62

[3] Yang, Y., Zhang, Y.L., Zhang, Z.C., et al. (2017) Study on the Relationship between Syndrome Elements and Homocysteine in High Risk Population of Stroke with Carotid Atherosclerosis. Chinese Journal of Traditional Chinese Medicine, 32, 537-541.

[4] Liu, D., Ao, W., Xu, M., et al. (2016) Significance of Detection of Serum Hcy, $\beta$-2MG and hs-CRP in Patients with H-Type Hypertension and Coronary Heart Disease. Chinese Journal of Evidence-Based Cardiovascular Medicine, 8, 687-690.

[5] Zhang, X.Z., Liu, F.Y., Li, J., et al. (2015) Correlation between Vascular Endothelial Function and Homocysteine in Elderly Patients with Coronary Heart Disease. Chinese Journal of Cardiovascular and Cerebrovascular Diseases in the Elderly, 17, 1268-1270.

[6] Yan, G.L., Wang, D., Qiao, Y., et al. (2015) Effect of Hyperhomocysteine Level on Severity of Coronary Heart Disease and Prognosis of Stent Implantation. Chinese Journal of Cardiovascular Diseases, 43, 500. 
[7] Yi, M.Y., Tan, J.H., Zhang, A.A., Li, F.J. and Liu, Y.J. (2018) Research Progress of Homocysteine and Atherosclerosis Mechanism. Journal of Hebei North University (Natural Science Edition), 34, 56-60.

[8] Huang, J.M., Jian, C.D., Tang, X.L., et al. (2011) Study on the Relationship between Hyperhomocysteinemia and Oxidative Stress and Inflammatory Response in Elderly Patients with Cerebral Infarction. Chinese Journal of Cardiovascular and Cerebrovascular Diseases in the Elderly, 13, 249-251.

[9] Wang, H.Y. (2012) Changes of Serum Homocysteine and Oxidative Stress in Patients with Diabetic Nephropathy. Guide to Chinese Medicine, 14, 1408-1409.

[10] Park, C.S., Shen, Y., Lewis, A., et al. (2016) Role of the Reprogramming Factor KLF4 in Blood Formation. Journal of Leukocyte Biology, 99, 673-685. https://doi.org/10.1189/jlb.1RU1215-539R

[11] Yoshida, T. and Hayashi, M. (2014) Role of Krüppel-Like Factor 4 and Its Binding Proteins in Vascular Disease. Journal of Atherosclerosis and Thrombosis, 21, 402-413. https://doi.org/10.5551/jat.23044

[12] Luo, C.Y., Ding, J.W., Zheng, X.X., Zhou, T. and Zhang, Z.G. (2018) Research Progress of Zinc Finger-Like Transcription Factor 4 and Atherosclerosis. Chinese Journal of Gerontology, 38, 3290-3293.

[13] Zheng, B., Han, M., Wen, J.K., et al. (2010) Role of Krüppel-Like Factor 4 in Phenotypic Switching and Proliferation of Vascular Smooth Muscle Cells. IUBMB Life, 62, 132-139. https://doi.org/10.1002/iub.298

[14] Li, H. and Sun, L.D. (2018) New Progress in the Study of Anti-Aging and Antioxidant Pharmacological Mechanism of Salidroside. China Medical Guide, 15, 51-54+81.

[15] Zhang, Y.Y., Li, C., Huang, Y.P., Zhao, S., Xu, Y.N., Chen, Y., Jiang, F., Tao, L. and Shen, X.C. (2020) EOFAZ Inhibits Endothelial-to-Mesenchymal Transition through Downregulation of KLF4. International Journal of Molecular Medicine, 46, 300-310. https://doi.org/10.3892/ijmm.2020.4572

[16] Wu, H.Y., Wang, Y.Y., Dong, H.L., Wu, Y.F., Wang, G. and Dai, M. (2018) Effect of CARTPT on Hydrogen Peroxide-Induced Senescence of Human Umbilical Vein Endothelial Cells. Guangdong Medicine, 39, 2117-2121.

[17] Xu, Q. (2014) Study on the Role and Mechanism of Cell Senescence Induced by Transcription Factors KLF4 and DEC1 in Tumor. Peking Union Medical College, Beijing.

[18] Sun, L., Dou, F., Chen, J., et al. (2018) Salidroside Slows the Progression of EA.hy926 Cell Senescence by Regulating the Cell Cycle in an Atherosclerosis Model. Molecular Medicine Reports, 17, 257-263. https://doi.org/10.3892/mmr.2017.7872

[19] Mao, G.X., Wang, Y., Qiu, Q., et al. (2010) Salidroside Protects Human Fibroblast Cells from Premature Senescence Induced by $\mathrm{H}_{2} \mathrm{O}_{2}$ Partly through Modulating Oxidative Status. Mechanisms of Ageing and Development, 131, 723-731. https://doi.org/10.1016/j.mad.2010.10.003 